Cahiers

d'ethnomusicologie

\section{Cahiers d'ethnomusicologie}

Anciennement Cahiers de musiques traditionnelles

$19 \mid 2006$

Chamanisme et possession

\title{
Bodega, bodégaires! Anthologie de la cornemuse du
}

Haut-Languedoc

CLRMDT - CORDAE/La Talvera - Conservatoire occitan, 2004

\section{Michel Plisson}

\section{OpenEdition}

\section{Journals}

Édition électronique

URL : http://journals.openedition.org/ethnomusicologie/151

ISSN : 2235-7688

Éditeur

ADEM - Ateliers d'ethnomusicologie

Édition imprimée

Date de publication : 1 novembre 2006

Pagination : 290-291

ISSN : 1662-372X

Référence électronique

Michel Plisson, «Bodega, bodégaires! Anthologie de la cornemuse du Haut-Languedoc », Cahiers

d'ethnomusicologie [En ligne], 19 | 2006, mis en ligne le 15 janvier 2012, consulté le 20 avril 2019. URL : http://journals.openedition.org/ethnomusicologie/151

Ce document a été généré automatiquement le 20 avril 2019.

Tous droits réservés 


\title{
Bodega, bodégaires! Anthologie de la cornemuse du Haut-Languedoc
}

CLRMDT - CORDAE/La Talvera - Conservatoire occitan, 2004

\author{
Michel Plisson
}

\section{RÉFÉRENCE}

Bodega, bodégaires! Anthologie de la cornemuse du Haut-Languedoc. Sous la direction de Luc Charles-Dominique et Daniel Loddo. 3 CD + Un livret. CLRMDT - CORDAE/La Talvera Conservatoire occitan - ADDMD 11, 2004.

1 On n'hésite pas à qualifier cette production des meilleurs superlatifs tant on est enthousiaste devant ce magnifique travail dont la responsabilité éditoriale - tant de la forme que du contenu - incombe au Conservatoire occitan, à Luc Charles Dominique et au Centre Languedoc Roussillon des Musiques et Danses ainsi qu'à Daniel Loddo et son inlassable travail avec son association La Talvera, à Cordes sur Ciel, en plein cœur du pays occitan.

2 Un travail d'une telle ampleur, $3 \mathrm{CD}+$ un livret de 112 pages, ne pouvait se faire rapidement. C'est un labeur qui s'est étendue sur plusieurs années, voire beaucoup plus si l'on compte les dates de collectage effectué par D. Loddo. Dans ce travail, les auteurs ont intelligemment dosé les enregistrements issus d'archives sonores ( $1 \mathrm{CD}$ complet) et les pratiques vivantes $(2 \mathrm{CD})$ de musiciens actuels qui démontrent que le lien de la tradition n'a pas été rompu. De fait, pour L.C. Dominique, cette publication est surtout « le reflet d'une pratique vivante, avec des musiciens actuels, de plein pied avec une démarche artistique et culturelle contemporaine ».

3 La bodega, appelée encore craba (chèvre en occitan), c'est la grande cornemuse du Haut Languedoc à un seul et long bourdon reposant sur l'épaule du musicien et terminé par une anche simple battante. Le graile (hautbois) quant à lui, est muni d'une anche double et se joue parfois tout seul. Si l'on sait qu'il existait plusieurs centaines de joueurs de bodega 
au début du XIX ${ }^{e}$ siècle dans la seule région autour de Castres, on a, en revanche plus de difficultés à établir ses origines et son histoire. Le livre de Pierre Bec ${ }^{1}$ nous renseigne sur les très nombreuses occurrences organologiques de la cornemuse à travers l'histoire, qui sont, indubitablement, polymorphistes (plusieurs termes renvoyant au même instrument) et polysémantiques (un seul terme désignant en apparence plusieurs instruments), lesquelles caractéristiques «semblent en effet endémiques à cette époque ». Dans l'espace et dans le temps on trouve ainsi une bonne trentaine de termes dont on peut penser qu'ils désignent le même type instrument ${ }^{2}$. Cela dénote pour le moins sa grande popularité à certains moments de l'histoire. Pour la bodega elle-même, le terme vient de la racine bot-/ ou bod qui signifie toute chose enflée. Malgré ces lumières lexicologiques et étymologiques, on sait beaucoup moins de choses sur son introduction en Languedoc et sur ses pratiques sociales. Le point non discuté est son ancienneté. On trouve en effet des traces occitanes à partir du XIV ${ }^{\mathrm{e}}$ siècle (Carcassonne), sous formes de sculptures, peintures, bas reliefs, vitraux dans divers châteaux et églises... Les traces sont plus nombreuses à partir du XVIII ${ }^{e}$ siècle et surtout du XIX ${ }^{e}$ siècle où son aire de diffusion semble s'accroître en proportion directe de l'émigration paysanne. Quoiqu'il en soit, il est avéré que jusqu'en 1914 presque chaque village avaient ses bodégaires et crabaires qui participaient «à la plupart des fêtes, danses et veillées " ${ }^{3}$ espace musical que la grande cornemuse partageait avec d'autres instruments comme les grailes, violons, flûtes à bec, tambours à friction, accordéons... Quasi-disparue dans les années trente, la bodega réapparut grâce à quelques passionnés comme Charles Alexandre (que l'on peut entendre dans le $\mathrm{CD}^{\circ}{ }^{\circ}$ ) qui depuis ont fait école. Aujourd'hui, on compte plusieurs dizaines de joueurs de bodega dans le sud de la France.

4 Le premier $\mathrm{CD}$ contient des enregistrements historiques ainsi que du collectage de l'association La Talvera effectué dans les années 1980-1990 qui concerne la bodega mais aussi des chants a cappella et de la musique d'accordéon. Les deux autres CD contiennent des enregistrements de groupes actuels dont certains s'inscrivent dans la tradition et d'autres moins. Les prises de son sont très bonnes et le choix des pièces montre une grande diversité de répertoire. Au final, un remarquable album à caractère anthologique.

\section{NOTES}

1. Pierre BEC, 1996, La cornemuse/Sens et histoire de ses désignations/Poésie, musique, folklore... par Pierre Bec. Isatis/Cahiers d'ethnomusicologie régionale $n^{\circ} 4 /$ Conservatoire occitan/Centre des Musiques et Danses Traditionnelles en Midi-Pyrénées.

2. En petite Catalogue, Pierre Bec trouve pas moins de 17 termes lexico-organologiques différents, Bec, idem, p. 35.

3. Bec, idem, p. 40. 